\title{
Single fibre electromyography in multifocal motor neuropathy with persistent conduction blocks
}

\author{
A Lagueny, G Le Masson, P Burbeaud, Ph Deliac
}

\begin{abstract}
Objective-To study the process of denervation-reinnervation in multifocal motor neuropathy with persistent conduction blocks in clinically affected and unaffected muscles.

Method-Volitional single fibre electromyography (SFEMG) was performed in the extensor digitorum communis (EDC) of seven patients. The jitter, the fibre density, and the mean interpotential interval were determined. The results before and after treatment with intravenous immunoglobulin (IVIg) between the unaffected EDC and affected EDC examined during the same SFEMG session were also compared. In addition the values of jitter, fibre density, and mean interpotential interval were analysed for correlation with the strength score on the MRC scale, the duration of the neuropathy, the number of IVIg treatment periods, and the radial nerve conduction block values.

Results-Mean jitter, percentage of jitters $>60 \mu \mathrm{s}$, and impulse blocking percentage, were higher than normal in both the affected EDCs and to a lesser degree in unaffected EDCs. Jitter decreased significantly after IVIg and correlated only with the MRC score. Fibre density and mean interpotential interval were higher than normal equally in the affected EDC and unaffected EDCs, but no correlation was found with strength, duration of the neuropathy, number of treatment periods, and conduction block values.
\end{abstract}

Conclusion-The major finding is the presence of SFEMG abnormalities in clinically unaffected EDCs. This shows a process of denervation-reinnervation even in the absence of clinical symptoms, probably more frequent than commonly supposed in this neuropathy. The rapid clinical improvement after IVIg infusions could be due to remyelination after demyelination and to an interference of IVIg with the blocking effect of antibodies on the $\mathrm{Na}^{+}$channels at the motor nerve endings.

(F Neurol Neurosurg Psychiatry 1998;65:357-361)

Keywords: multifocal motor neuropathy; single fibre electromyography

Dr A Lagueny, Service

Neurologie, Hopital du

Haut-Leveque, USN, Avenue

de Magellan, 33604 Pessac,

France. Telephone 0033556

420; fax 0033556815 .

Received 23 September 1997 and in revised form 25

November 1997

Accepted 19 February 1998 conduction blocks along the motor nerves outside the areas of possible compression. ${ }^{2}$ Multifocal motor neuropathy with persistent conduction blocks is considered an immune mediated demyelinating neuropathy. ${ }^{3}$ Experimental conduction blocks can be induced using the serum of patients with high titres of circulating antibodies to $\mathrm{GM} 1,{ }^{4-6}$ although the link between the motor conduction blocks and the anti-GM1 antibodies has not been definitely established. ${ }^{78}$ Weakness improves rapidly, although often temporarily, ${ }^{9}{ }^{10}$ with high doses of intravenous immunoglobulin (IVIg). ${ }^{11-13}$ On the other hand, some patients who are less responsive to $\operatorname{IVIg}^{14}$ develop atrophy of the muscles innervated by the nerves involved in the dysimmune demyelinating process. In more severe forms, the neuropathy may mimic an anterior horn cell disease. ${ }^{15}$ In these cases, standard EMG may detect signs of denervation with fibrillation potentials, and signs of reinnervation with giant motor unit action potentials (MUAPs), suggesting an extension of the territory of the motor units, or at least an increase of fibre density in the uptake area of the coaxial needle. A means of investigating the process of denervationreinnervation during the course of a neurogenic involvement is to use single fibre electromyography (SFEMG), ${ }^{16}{ }^{17}$ and this was used here to study the functional status of the motor units during the course of the multifocal motor neuropathy with persistent conduction blocks.

\section{Patients and methods}

PATIENTS

Seven patients with an involvement of the extensors at the forearm, unilateral in four and bilateral in three, were included in the study, the extensor digitorum communis (EDC) being chosen for SFEMG examination. All patients had developed an asymmetric motor neuropathy beginning in the upper limbs, with a progressive deterioration in four and a stepwise deterioration in three. The time between the presumed onset of the neuropathy and the electrophysiological diagnosis ranged from 10 to 60 months. No patient displayed signs of upper motor neuron or bulbar involvement. Sensory examination was normal in all the patients, although one complained of paraesthesia in the ulnar area, and another of diffuse pain in the right hand but which had disappeared when the weakness appeared. A distal atrophy of the upper limbs was found in three patients, bilateral and slight in two and unilateral but more marked in the other. Protein concentration in CSF was within the normal limit in all the patients, and anti-GM1 
Table 1 Demographic data

\begin{tabular}{|c|c|c|c|c|c|c|c|}
\hline Patients & P1 & $P 2$ & P3 & P4 & P5 & P6 & P7 \\
\hline Age/sex & $44 / \mathrm{M}$ & $37 / \mathrm{M}$ & $40 / \mathrm{F}$ & $65 / M$ & $60 / \mathrm{M}$ & $47 / \mathrm{F}$ & $41 / \mathrm{M}$ \\
\hline Duration (months) & 10 & 25 & 14 & 12 & 60 & 10 & 19 \\
\hline Progression & Stepwise & Progressive & Progressive & Progressive & Stepwise & Stepwise & Stepwise \\
\hline Weakness & Upper limbs & Upper limbs & Upper limb & Upper limb & Upper limbs & Upper+lower limbs & Upper limb \\
\hline \multirow[t]{2}{*}{ Location } & Bilateral & Bilateral & Right & Right & Bilateral & Right & Left \\
\hline & Distal+proximal & Distal & Distal & Distal & Distal & Distal & Distal \\
\hline Atrophy & None & Hands & None & None & Hand & None & Hands (bilateral) \\
\hline Location & - & Bilateral & - & - & Left & - & Forearm (left) \\
\hline AntiGM1 titre & 3200 & ND & ND & ND & 800 & 800 & 800 \\
\hline CTx & No & Yes & No & No & No & No & Yes \\
\hline
\end{tabular}

Duration=time elapsed between onset of symptoms and diagnosis; ND=Not detected; $\mathrm{Ctx}=$ cyclophosphamide.

antibody titres measured by enzyme linked immunosorbent assay (ELISA) were significantly high in four of the seven. Once diagnosis was made, treatment with IVIg $(0.4 \mathrm{~g} / \mathrm{kg} /$ day for 5 days) was given, which improved strength temporarily. When renewed, treatment usually provided the same effect. Additional treatment with oral cyclophosphamide (CTx: $2 \mathrm{mg} / \mathrm{kg}$ ) day) was given in two patients who were more severely affected. The table provides clinical and biological data at diagnosis including the titres of anti-GM1 antibodies when significantly positive $(>20)$.

METHOD

Electrodiagnosis was performed using Dantec Counterpoint MKII apparatus.

Determination of the conduction block

Surface electrodes were used for stimulating the nerves and recording the compound muscle action potentials (CMAPs). A conduction block was considered when the CMAP amplitude and the negative phase area on proximal stimulation was at least $50 \%$ smaller, with a negative peak duration at the most $15 \%$ longer, than on distal stimulation. In these cases attempts were made to determine the shortest measurable limb segment, to reduce the influence of conduction dispersion and phase cancellation on the negative phase area. ${ }^{18} 19$ The sites of supramaximal stimulation were for $(a)$ the median nerve with recording from the abductor pollicis brevis: wrist, elbow, axilla, and Erb's point ; $(b)$ the ulnar nerve with recording from the abductor digiti minimi: wrist, below elbow, above elbow, axilla, and Erb's point; $(c)$ the radial nerve with recording from the extensor indicis: elbow, radial groove, and posterior cord of the brachial plexus, $(d)$ the tibial nerve with recording from the abductor hallucis: ankle, and popliteal fossa; and (e) the peroneal nerve with recording from the extensor digitorum brevis: ankle, below the fibula, and above the fibula.

In addition to motor nerve conductions, routine electrodiagnosis included: concentric needle EMG (but in which amplitude, duration, and number of phases of individual MUAPs were not systematically analysed) and antidromic sensory nerve conductions from the superficial sensory radial and sural nerves. Conduction studies were performed at a skin temperature of $32^{\circ} \mathrm{C}$.
Single fibre electromyography

Single fibre electromyography was performed using the SFEMG programme of the Counterpoint and a Nicolet SFEMG electrode (length $40 \mathrm{~mm}$ ). Volitional SFEMG was chosen because it is more suitable than stimulated SFEMG for studying fibre density. The EDC was examined when the MRC score was at least 3 to permit recording of an adequate number of muscle fibre action potentials in various motor units to provide the fibre density and mean interpotential interval. The time between the onset of symptoms and the first SFEMG examination varied from 15 to 54 months. The examinations were performed before the perfusions of IVIg and during the period of efficiency of IVIg, as all patients were good responders. In one patient we were able to use this procedure five times over a period of 50 months; we used it four times in one other and three times in the remaining five. From one session to the next, various parts of the EDC were explored to minimise the risk of recording in areas damaged by the SFEMG needle during the previous session. In the four patients with unilateral involvement, we examined the opposite EDC, which had never been involved clinically. The high pass filter was set at 500 $\mathrm{Hz}$, the amplifier gain between $200 \mu \mathrm{V}$ and 1 $\mathrm{mV}$, and the sweep speed between 0.5 and 5 $\mathrm{ms} /$ division, depending on the amplitude of the muscle action potentials and the duration of the complexes formed by the muscle fibre action potentials of each motor unit in the uptake area of the SFEMG electrode. More than 20 different sites from the EDC were investigated. Action potentials with amplitudes $>200 \mu \mathrm{V}$ and rise times $<300 \mu$ s were selected for calculating fibre density and jitter. The mean value of consecutive differences over 100 discharges from each action potential pair, and the mean jitter with SD, were calculated by the SFEMG software of the Counterpoint from 20 pairs with an IPI $<4$ ms. The mean interpotential interval was calculated with the formula:

mean interpotential interval $=\sum_{N}^{1}\left[\frac{d}{n-1}\right] / N$

where $\mathrm{N}$ is the number of the complexes studied, $d$ the duration between the negative peaks of the first and last action potentials, and $\mathrm{n}$ the number of action potentials. The upper limits of normal values, taking into account the age of the patients, are those of multicentre 
Before IV Ig After IV Ig
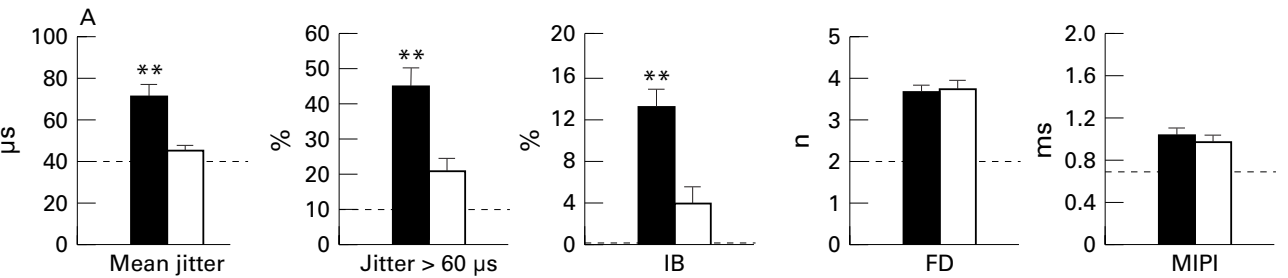

Affected EDC

Unaffected EDC
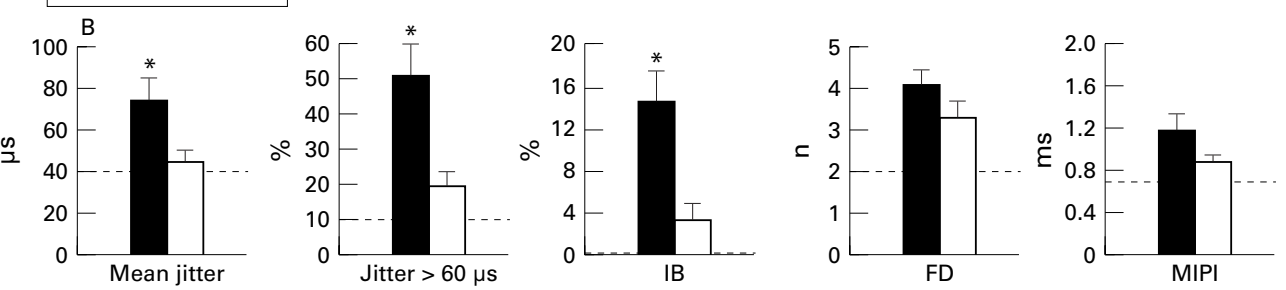

Figure 1 (A) Comparison of the SFEMG indices: mean jitter, percentage of jitter $>60 \mu$ s, percentage of impulse blocking (IB), fibre density (FD), and mean interpotential interval (MIPI) in the affected extensor digitorum communis(EDCs) between, before, and after IVIg. All values are abnormal. Mean jitter, percentage of jitters $>60 \mu$, and percentage of IB decreased significantly on IVIg, but not fibre density and mean interpotential interval. ${ }^{\star} p<0.01 ;{ }^{\star} p<0.05$. Upper normal limit is indicated by the dotted line. (B) Comparison of the SFEMG indices between affected and unaffected EDCs examined at the same session. The values are also abnormal in the unaffected EDCs. Mean jitter, percentage of jitter $>60 \mu$ s, and percentage of IB were significantly lower in unaffected EDC than in affected EDC, but fibre density and mean interpotential interval were not. ${ }^{*} p<0.05$. Upper normal limit is indicated by the dotted line.

studies $^{16}{ }^{17}$ : fibre density $<2$, mean jitter $<40$ with no more than $10 \%$ of MCD values $>60 \mu \mathrm{s}$, and mean interpotential interval $\leqslant 0.70$.

\section{STATISTICS}

Using the Mann-Whitney test we compared the values of the SFEMG indices: fibre density, mean jitter, percentage of jitter $>60 \mu$ s, the proportion of pairs blocked (impulse blocking percentage), and mean interpotential interval, obtained before IVIg and during the effect of IVIg. We also compared the results of the SFEMG indices on the affected and unaffected sides examined during the same session. Using the Spearman rank test, we also searched for correlations between the SFEMG indices and the following: time elapsed between the onset of symptoms and the SFEMG session, the number of IVIg courses, the MRC score of the EDC examined, and the degree of the conduction block (conduction block percentage) in the radial nerve evaluated at each session.

\section{Results}

CONDUCTION BLOCKS

Conduction blocks were found in at least three nerves, including the radial nerve, in all the patients except one where blocks were found in the ulnar and median nerves but not in the radial nerve. However, it was probable that the weakness of the EDC in this patient resulted from proximal or distal conduction blocks in the radial nerve which were not detected by the method used. A low CMAP amplitude after distal stimulation, resulting either from a distal conduction block or axonal loss, was found in three patients.

STANDARD EMG

Standard EMG showed reduced recruitment with some large potentials in the affected EDC, and normal patterns in the unaffected EDC at diagnosis, but standard EMG was not performed at the SFEMG sessions. Antidromic sensory nerve conductions were normal.

MEAN VALUES OF THE SFEMG INDICES

The mean values of the SFEMG indices were all abnormal. On the affected side, mean jitter was $73 \mu$ s before treatment and decreased significantly on IVIg to $44 \mu$ s, which was however above the normal upper limit; the percentage of jitter $>60 \mu$ s and the impulse blocking percentage, respectively $44 \%$ and $13 \%$ before treatment, also decreased significantly on IVIg, to $20 \%$ and $4 \%$ respectively. On the other hand fibre density and mean interpotential interval, respectively 3.5 and 1.02 before treatment, did not change significantly (respectively 3.6 and 0.96) after IVIg (fig 1A). The SFEMG indices were also all abnormal in the EDC on the clinically unaffected side. However, on the unaffected side the mean jitter $(45 \mu \mathrm{s})$, the percentage of jitter $>60 \mu \mathrm{s}(19.2 \%)$, and the impulse blocking percentage $(3.2 \%)$ were significantly lower than on the affected side examined at the same session $(74 \mu \mathrm{s}, 50.6 \%$, and $14.6 \%$ respectively). On the other hand the values of fibre density and mean interpotential interval were not statistically different between the unaffected side (3.2 and 0.88) and the affected side (4.0 and 1.18) (fig 1B). In only one patient the unaffected EDC displayed normal mean jitter but with abnormal fibre density and abnormal mean interpotential interval. Another clinically unaffected EDC, displaying abnormal SFEMG indices at examination, later became clinically affected.

As jitter decreased on IVIg, it was not surprising to find a strong correlation between the MRC score and the following indices: mean jitter $(r=0.674, \mathrm{p}=0.0001)$, percentage of 

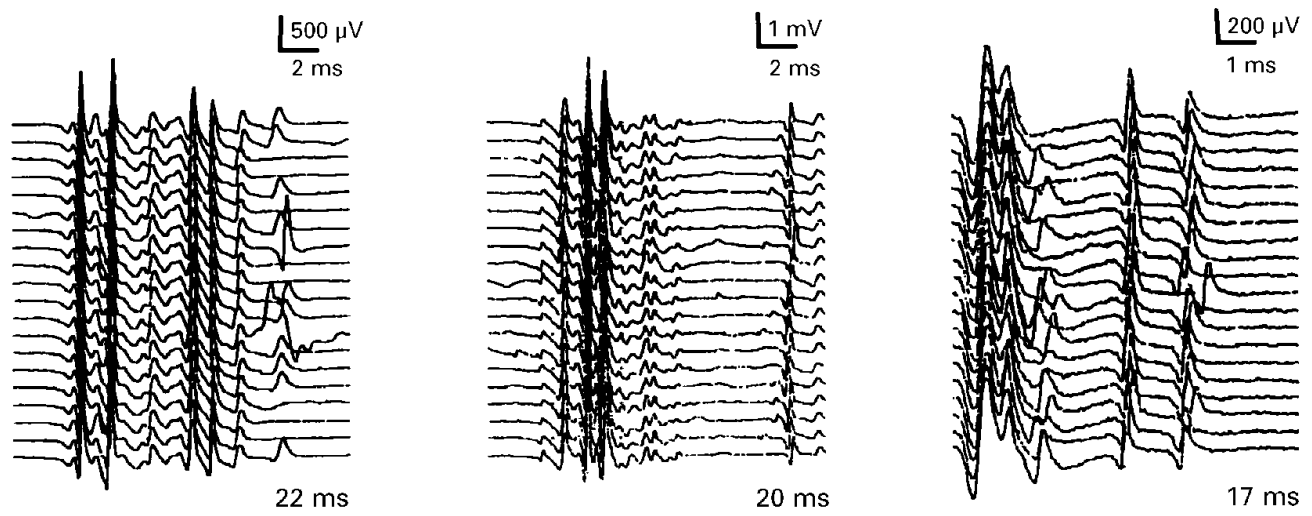

Figure 2 Three examples of SFEMG from the extensor digitorum communis. Twenty consecutive discharges of three complexes with increased jitters and long interpotential intervals are shown.

jitter $>60 \%(r=0.669, \mathrm{p}=0.0009)$, and impulse blocking percentage $(r=0.676, \mathrm{p}=0.0005)$; the lower the MRC score, the higher the jitter. On the other hand, jitter, fibre density, and mean interpotential interval were not correlated with the time elapsed between the onset of symptoms and the SFEMG examinations, the number of IVIg courses, and the conduction block percentage.

\section{Discussion}

In peripheral neuropathy, lesions of the motor axons result in a process of denervationreinnervation which is responsible for changes in the functional status of the motor units investigated by SFEMG. ${ }^{16}{ }^{17}$ These changes include increased fibre density (a marker of reinnervation through axonal sprouting), longer interpotential interval increasing with length of collateral sproutings, and increased jitter with blocking in the newly formed neuromuscular junctions during the process of reinnervation. Abnormal jitters and impulse blockings are numerous in rapidly evolving denervations as a result of abnormal neuromuscular transmission in immature end plates. To a lesser extent disturbed conductions along the nerve twigs and muscle fibres may also increase jitter.

Jitter, fibre density, and mean interpotential interval were abnormal in the EDC of seven patients with multifocal motor neuropathy with persistent conduction blocks in both clinically affected and unaffected EDCs, indicating a process of denervation-reinnervation even in the clinically unaffected EDC (fig 2). In neuromuscular diseases jitter is dependent on the motor unit discharge rate. It could be deduced, therefore, that the decrease of jitter in the affected EDC after IVIg may result only from the decrease of motor unit discharge. However, the discharge rate was monitored by a rate meter, and maintained by our patients at roughly the same level whatever the MRC score. We therefore postulate that the decrease of the mean jitter resulted directly from the IVIg infusions which improved strength in affected muscles, and not from a decrease of the motor unit discharge rate. On treatment, as jitter was diminishing, the complexes became more stable than during a relapse, although the jitter usually remained above the upper limit of the normal even when the strength became normal again. However, no correlation was found between the values of jitter and the total number of infusions, confirming the clinically non-cumulative effect of the infusions.

Infusion of IVIg is effective within a week in up to $90 \%$ of patients with multifocal motor neuropathy with persistent conduction blocks. It could interfere with antibody-antigen binding at the nodes of Ranvier. ${ }^{1}$ The rapid clinical improvement is usually accompanied by a reduction of conduction blocks, or an increase in the distal CMAP amplitude. ${ }^{20}$ In the rat, one mechanism underlying amelioration of strength and conduction block after a focal demyelinative lesion produced by intraneural injection of antiserum from rabbits is remyelination. This has been reported to appear 8 days after the injection. ${ }^{21}$ However, another mechanism may be implicated. Conduction blocks have been induced by anti-GM1 antibodies which block the $\mathrm{Na}^{+}$channels in the presence of complement. ${ }^{22}$ Moreover, the serum of patients with multifocal motor neuropathy with or without anti-GM1 antibodies has been found to induce a rapid reduction of the end plate potential at the neuromuscular junction. The mechanism is not an interference with acetylcholine release but more probably a blockade of the $\mathrm{Na}^{+}$current in the distal part of the motor nerve and consequently of the action potential electrogenesis. ${ }^{23}$ Failure of the release of acetylcholine due to the immunological blockade of the $\mathrm{Na}^{+}$channels in the distal part of the motor nerve, ${ }^{24}$ rapidly reversed by IVIg which interferes with this blockade, could explain why jitter increased during the relapses before IVIg in our patients, and decreased after infusion as strength improved.

Fibre density and mean interpotential interval were also abnormal, even in the clinically unaffected EDCs. Unlike jitter, the fibre density and mean interpotential interval values did not correlate with the effect of the IVIg. Multifocal motor neuropathy with persistent conduction blocks is a chronic neuropathy in which the immunological process inhibits 
remyelination and reorganisation of $\mathrm{Na}^{+}$channels, and can damage the axonal membrane. ${ }^{25}{ }^{26}$ The axonal damage is responsible for atrophy appearing in some cases. The more severe forms of the neuropathy may mimic a lower motor neuron disease with large MUAPs and late components on standard EMG. ${ }^{27}$ Loss of spinal motor neurons, probably by retrograde degeneration from axonal damage at the roots, has been demonstrated in a neuropathological study of one case. ${ }^{28}$

In axonal damage without atrophy, SFEMG is a means of detecting a slight chronic involvement accompanied by changes in the functional status of the motor units. ${ }^{16}{ }^{17}$ The finding of increased fibre density and increased mean interpotential interval in clinically unaffected EDC where coaxial needle examination was normal at diagnosis, is interesting. It shows that the axonal damage may be present before the occurrence of clinical signs. However, the jitter in the unaffected EDC was much less abnormal than in the controlateral affected EDC examined during the same session, suggesting that collateral reinnervation with a sufficient number of mature end plates may be one of the compensatory mechanisms explaining the normal strength in some muscles in multifocal motor neuropathy independently of whether the lesions of the motor axons are primary or secondary to demyelination.

\section{Conclusion}

The results of our SFEMG study in multifocal motor neuropathy with persistent conduction blocks suggest a dual mechanism for the abnormalities found. One could be due to the blocking effect of antibodies on the $\mathrm{Na}^{+}$ channels, ${ }^{29}$ rapidly reversible using IVIg infusion and probably accounting for the correlation between the jitter and the score of the MRC rating scale. The other, slower mechanism, could be due to a denervationreinnervation process, with increased fibre density, increased mean interpotential interval, and also increased jitter, the abnormality of which depends on the severity of the axonal involvement and the number of immature end plates. This process is probably more frequent than supposed in this neuropathy.

1 Nobile-Orazio E. Multifocal motor neuropathy. $\mathcal{F}$ Neurol Neurosurg Psychiatry 1996;60:599-603.

2 Krarup C, Stewart JD, Summer AJ, et al. A syndrome of asymmetric limb weakness with motor conduction block. Neurology 1990;40:118-27.

3 Parry G. Multifocal motor neuropathy. Muscle Nerve 1996; 19:269-76.

4 Santoro M, Uncini A, Corbo M, et al. Experimental conduction block induced by serum from a patient with anti-GM ${ }_{1}$ antibodies. Ann Neurol 1992;31:385-90.
5 Arasaki K, Kusunoki S, Kudo N, et al. Acute conduction block in vitro following exposure to antiganglioside sera. Muscle Nerve 1993;16:587-93.

6 Uncini A, Santoro M, Corbo M, et al. Conduction abnormalities induced by sera of patients with multifocal motor neuropathy and anti-GM $\mathrm{GM}_{1}$ antibodies. Muscle Nerve 1993;16:610-5

7 Lange DJ, Trojaborg W. Do GM $\mathrm{GM}_{1}$ antibodies induce demyelination? Muscle Nerve 1994;17:105-7.

8 Parry G. Antiganglioside antibodies do not necessarily play a role in multifocal motor neuropathy. Muscle Nerve 1994; 17:97-9.

9 Chaudhry V, Corse AM, Cornblath DR, et al. Multifocal motor neuropathy response to human immune globulin. Ann Neurol 1993;33:237-42.

10 Elliott JL, Pestronk A. Progression of multifocal motor neuropathy during apparently successful treatment with human immunoglobulin. Neurology 1994;44:967-8.

11 Nobile-Orazio E, Meucci N, Barbieri S, et al. High-dose intravenous imunoglobulin therapy in multifocal motor neuropathy. Neurology 1993;43:537-44.

12 Azulay JP, Blin O, Pouget J, et al. Intravenous immunoglobulin treatment in patients with motor neuron syndromes associated with anti-GM $\mathrm{GM}_{1}$ antibodies: a double blind, placebo-controlled study. Neurology 1994;44:42932.

13 Van den Berg LH, Kerkhoff H, Oey PL, et al. Treatment of multifocal motor neuropathy with high dose intravenous immunoglogulin: a double blind, placebo controlled study. 7 Neurol Neurosurg Psychiatry 1995;59:248-52.

14 Bouche $\mathrm{P}$, Moulonguet A, Younes-Chennoufi $\mathrm{AB}$, et al. Multifocal motor neuropathy with conduction block: a study of 24 patients. 7 Neurol Neurosurg Psychiatry 1995;59: 38-44.

15 Kaji R, Shibasaki H, Kimura J. Multifocal demyelinating motor neuropathy: cranial nerve involvement and immunoglobulin therapy. Neurology 1992;42:506-9.

16 Stålberg E, Trontelj JV. Single fiber electromyography. Studies in healthy and diseased muscle. 2nd ed. New York: Raven Press, 1994 .

17 Sanders DB, Stalberg EV. Single-fiber electromyography. Muscle Nerve 1996;19:1069-83.

18 Rhee EK, England JD, Summer AJ. A computer simulation of conduction block: effects produced by actual block versus interphase cancellation. Ann Neurol 1990;28:146-56.

19 Cornblath DR, Sumner AJ, Daube J, et al. Conduction block in clinical practice. Muscle Nerve 1991;14:869-71.

20 Chaudhry V, Corse AM, Cornblath DR, et al. Multifocal motor neuropathy: electrodiagnostic features. Muscle Nerve 1994;17:198-205.

21 Saida K, Sumner AJ, Takahiko S, et al. Antiserum-mediated demyelination: relationship between remyelination and functional recovery. Ann Neurol 1980;8:12-4.

22 Takigawa T, Yasuda H, Kikkawa R, et al. Antibodies against $\mathrm{GM}_{1}$ ganglioside affect $\mathrm{K}+$ and $\mathrm{Na}+$ currents in isolated rat myelinated nerve fibers. Ann Neurol 1995;37:436-42.

23 Roberts M, Willison HJ, Vincent A, et al. Multifocal motor neuropathy human sera block distal motor nerve conduction in mice. Ann Neurol 1995;38:111-8.

24 Waxman G. Sodium channel blockade by antibodies: a new mechanism of neurological disease ? Ann Neurol 1995;37: $421-3$.

25 Kaji R, Oka N, Tsuji T, et al. Pathological findings at the site of conduction block in multifocal motor neuropathy. Ann Neurol 1993;33:152-8.

26 Kaji R, Hirota N, Oka N, et al. Anti-GM $\mathrm{GM}_{1}$ antibodies and impaired blood-nerve barrier may interfere with remyelination in multifocal motor neuropathy. Muscle Nerve 1994;17:108-10.

27 Cruz Martinez A, Arpa J, Lara M. Electrophysiological improvement after intravenous immunoglobulin in motor neuropathy with multifocal conduction block. I Neurol Neurosurg Psychiatry 1993;56:1236-7.

28 Adams D, Kuntzer T, Steck AJ, et al. Motor conduction block and high titres of anti-GM ${ }_{1}$ ganglioside antibodies: pathological evidence of a motor neuropathy in a patient with lower motor neuron syndrome. $f$ Neurol Neurosurg Psychiatry 1993;56:982-7.

29 Gutmann L, Gutmann L. Axonal channelopathies: an evolving concept in the pathogenesis of peripheral nerve disorders. Neurology 1996;47:18-21. 